\title{
Conformations of ribonucleoside uridine in the low temperature Ar matrices
}

\author{
A.Yu. Ivanov \\ B. Verkin Institute for Low Temperature Physics and Engineering of the National Academy of Sciences of Ukraine \\ 47 Lenin Ave., Kharkov 61103, Ukraine \\ E-mail: ivanov@ilt.kharkov.ua \\ Received February 8, 2010
}

\begin{abstract}
The conformational equilibrium of the ribonucleoside uridine molecules in the low temperature Ar matrices was investigated by the FTIR matrix isolation spectroscopy and quantum-chemical calculations at the DFT and MP2 levels of theory. It was established that conformers with the carbonyl group $\mathrm{C} 2 \mathrm{O}$ which takes part in the intramolecular hydrogen bonds dominate in the low temperature matrices. Populations of syn- and anticonformers with intramolecular hydrogen bonds $\mathrm{O} 5{ }^{\prime} \mathrm{H}-\mathrm{O} 2$ and $\mathrm{O} 2{ }^{\prime}-\mathrm{O} 2$, respectively, are equal practically. Unlike 2'-deoxyuridine, the syn-conformers of Ur with C3'-endo structure of the ribose ring (South) have been found in Ar matrices. At the same time the conformations of $\mathrm{C}^{2}$ '-endo (North) ribose ring prevail both in antiand syn-conformers.
\end{abstract}

PACS: 33.15.-e Properties of molecules; 67.80. -s Quantum solids.

Keywords: FTIR-spectroscopy, low temperature matrix isolation, nucleosides.

Low temperature spectral methods play a significant role in the modern studies on structures of isolated fragments of biopolymer molecules (RNA, DNA, proteins) [1-5]. To isolate molecules from strong intermolecular interactions, supersonic beams of inert gases [1-3], nanodroplets (nanomatrices) of superfluid ${ }^{4} \mathrm{He}[4,5]$ or the classic method for matrix isolation in films of rare gases [6] are applied.

The present work continues investigations of nucleosides by FTIR matrix isolation spectroscopy [7-11]. The paper considers features of the pyrimidine ribonucleoside uridine (Ur) in the isolated state. Uridine is a structural element of ribonucleic acid (RNA), the molecule which could play a key role to the advent of life on Earth [12]. Up to the present, information on the uridine molecule structure was limited with experimental results on solutions and crystals as well as with quantum-chemical calculations [13-18]. Hitherto, we obtained some information on separate fragments of ribonucleoside uridine (Ur) spectrum [7], which showed the absence of thermal decomposition upon sample sputtering from Knudsen cell, according to our method, and the presence of characteristic bands of intramolecular hydrogen bonds in the spectrum. However, later on, the special attention has been concentrated on researching a simpler molecule, namely, 2'-deoxyuridine (dU) [8-11]. Unlike dU, Ur molecule includes the additional hydroxyl $\mathrm{O} 2 \mathrm{H}$ group. Due to this group, the more complicated structures of intramolecular hydrogen bonds can be revealed (Fig. 1).

For these work FTIR spectra of Ur molecules isolated in $\mathrm{Ar}$ matrices at various experimental conditions were obtained and compared with the spectra of dU in Ar matrices. UV irradiation and matrix annealing were applied to change of population of conformers fixed in the matrices. The comparison of experimental data and the results of calculations for the isolated molecules of Ur were carried out with the use of more precise quantum-chemical calculations, as compared to methods used before [14-18].

\section{Experimental and computational methods}

The key details of low-temperature experiments were described in some previous papers [19-21]. For this work FTIR spectra were obtained with apodized resolution $0.4 \mathrm{~cm}^{-1}$ in the $200-600 \mathrm{~cm}^{-1}, 450-2500 \mathrm{~cm}^{-1}$ and 1400 $4000 \mathrm{~cm}^{-1}$ ranges. Deposition of matrix on the low temperature mirror was controlled with the low temperature quartz microbalances $[19,21]$. The intensity of Ur molecular beam was within $60-70 \mathrm{ng} / \mathrm{s} \cdot \mathrm{cm}^{2}$, temperatures of evaporation from Knudsen cell being $420-430 \mathrm{~K}$. In this temperature range thermodestruction of Ur was not observed. The inert gas (Ar) purity was more than $99.99 \%$.

All quantum-chemical calculations were carried out using Firefly QC package (7.1.F version) [22] which is par- 

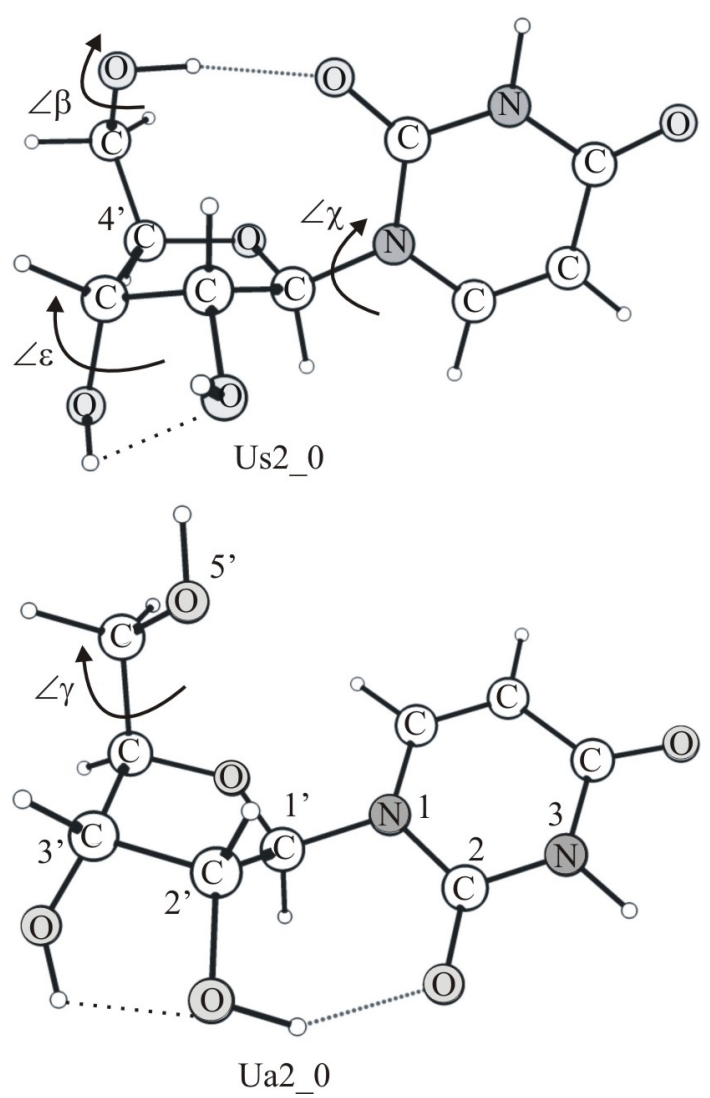

Fig. 1. The atom numbering, torsion angles and molecular structures of the main syn- (Us2_0) and anti- (Ua2_0) conformational isomers of ribonucleoside Ur. Intramolecular hydrogen bonds $\mathrm{O} 5$ ' $\mathrm{H}-\mathrm{O} 2$ are represented with dotted lines.

tially based on GAMESS (US) source code [23]. For the most part, our calculation were performed on the GRIDcluster of B. Verkin ILTPE and partially on the Core 2 Quad workstation. Standard capabilities of Firefly package were used for the estimations of Gibbs free energies $(\Delta G)$ of conformers. For Ur molecules sizes of correlation consistent basis sets cc-pVDZ, aug-cc-pVDZ and aug-ccpVTZ [24] were 315,533 and 1235 basis functions, respectively. The density functional theory (DFT) and Moller-Plesset perturbation theory (MP2 and MP4 levels) were used for our calculations. The fast and least expensive DFT/B3LYP/cc-pVDZ method was applied to calculate fragments of the map of potential energy surface. The most expensive calculations by MP2//aug-cc-pVTZ and MP4/ aug-cc-pVDZ//MP2/aug-cc-pVDZ methods were used only for estimating of the relative energies of the conformers fixed in matrices. Names of torsion angles C4'C5'O5'H $(\angle \beta), \mathrm{C}^{\prime}{ }^{\prime} \mathrm{C}^{\prime}{ }^{\prime} \mathrm{C} 5^{\prime} \mathrm{O} 5{ }^{\prime}(\angle \gamma), \mathrm{O}^{\prime}{ }^{\prime} \mathrm{C} 1{ }^{\prime} \mathrm{N} 1 \mathrm{C} 2$ $(\angle \chi), \mathrm{C}^{\prime}{ }^{\prime} \mathrm{C}^{\prime}{ }^{\prime} \mathrm{O} 3^{\prime} \mathrm{H}(\angle \varepsilon)$ and the method for calculating the angle of pseudorotation of the ribose ring $(\angle P)$ are given in accordance with Saenger's monograph [13]. Conformations with the pyrimidine ring anti-orientation [13] and syn-conformers [13] are denoted as Uan_ $x$ and Us $n \_x$ where $n$ shows ribose conformations (2-C2'-endo or 3-C $3^{-}$, -endo) and $x$ is the number ordered by increasing of the relative energies of conformations. For the correct measurements of spectral band intensities and comparisons of the experimental and calculated spectra, experimental spectra were approximated with Gaussian functions by the FITYK program [25].

\section{Results and discussion}

The experimental spectrum in the range of characteristic bands of $v \mathrm{OH}$ and $v \mathrm{NH}$ stretching vibrations of Ur conformers is presented in Fig. 2. For comparison, Fig. 2 shows the $\mathrm{dU}$ spectrum in Ar matrix. The addition of the hydroxyl group O2'H leads to appearing of two new groups of spectral bands (3584-3551 $\mathrm{cm}^{-1}$ and 3458-3449 $\mathrm{cm}^{-1}$ ) in the spectrum of Ur. Positions and intensities of these bands suggest the presence of the intramolecular hydrogen bonds $\mathrm{O} 2{ }^{\prime} \mathrm{H}-\mathrm{O} 2, \mathrm{O}^{\prime} \mathrm{H}-\mathrm{O} 2$ ' (Fig. 1) and $\mathrm{O}^{\prime}{ }^{\prime} \mathrm{H}-\mathrm{O} 3$ '. The most complete picture of possible intramolecular hydrogen bonds in the Ur conformations was obtained earlier by quantum-chemical calculations [18] but these results cannot be used directly for comparison with those of the matrix experiments. In particular, the result of the work [18] does not allow distinguishing the conformers isolated by high barriers. It is known that only a sufficiently large barrier between conformers prevents from the shift of conformational equilibrium (interconversion) upon matrix deposition from the gaseous phase [26,27]. Upon annealing of the already deposited matrix at $30 \mathrm{~K}$, the boundary height of the barrier is about $2.5-3 \mathrm{kcal} / \mathrm{mol}\left(\hbar v=1000 \mathrm{~cm}^{-1}\right)$ [27]. However, preventing interconversion in the process of cooling of hot molecules to the temperature of the matrix may require a higher barrier [10]. For example, results of

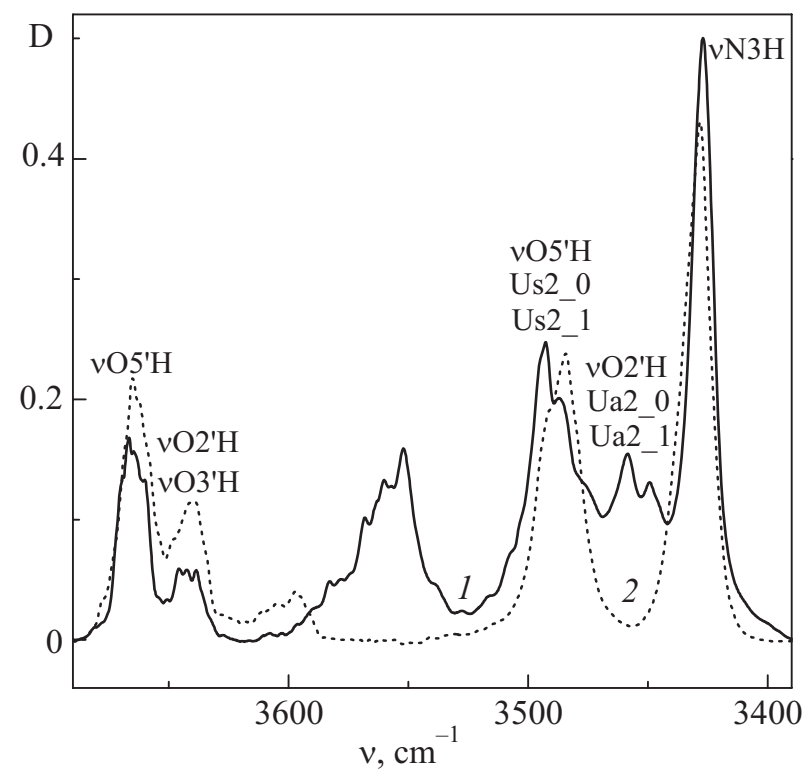

Fig. 2. The region of the $\mathrm{vOH}, \mathrm{vNH}$ stretching vibrations of the Ur (1) and dU (2) conformers. These spectra were brought to equal intensity of the $v \mathrm{~N} 3 \mathrm{H}$ bands. 
our previous work [11] showed that the hydroxyl group $\mathrm{O} 2$ ' $\mathrm{H}$ with $1.3 \mathrm{kcal} / \mathrm{mol}$ barrier may rotate during deposition into $\mathrm{Kr}$ matrix at $6 \mathrm{~K}$.

To estimate the heights of transition barriers in a family of anti-conformers Ua2_0 (Fig. 1) with intramolecular hydrogen bonds $\mathrm{O} 2^{\prime} \mathrm{H}-\mathrm{O} 2$ and $\mathrm{C}^{\prime}-$-endo structure of the ribose ring, the map of the potential energy surface in coordinates of $\chi$ and $\beta$ angles was constructed with step of $30^{\circ}$ (Fig. 3). Figure 3 demonstrates $\beta$ «grooves» three extended along the angle, separated by high barriers $(>3 \mathrm{kcal} / \mathrm{mol}$ ). Within these grooves the barriers are less than $2 \mathrm{kcal} / \mathrm{mol}$ and the probability of interconversion of conformers in the process of deposition in the matrix at a temperature of $12 \mathrm{~K}$ is high. The cross-section of the groove with Ua2_0 structure is shown in Fig. 4,a. As is seen, the height of transition barriers $\mathrm{Ua} 2 \_0 \mathrm{a} \rightarrow \mathrm{Ua} 2 \_0$ and $\mathrm{Ua} 2 \_\mathrm{b} \rightarrow \mathrm{Ua} 2 \_0$ do not exceed $0.7 \mathrm{kcal} / \mathrm{mol}$ as calculated on the DFT/cc-pVDZ level of theory. MP2/cc-pVDZ calculations predict even lower values of barriers $(<0.3 \mathrm{kcal} / \mathrm{mol})$. With these barriers the Ua2_0a and Ua2_0b conformers transform to Ua2_0 structure during deposition into Ar matrix at $12 \mathrm{~K}$. In a similar manner, the profile of the bottom of the groove with Ua2_1 and Ua2_2 structures (Fig. 4,b) predicts a transition Ua2_2 $\rightarrow \mathrm{Ua} 2 \_1$ during the matrix formation.

Formation of the intramolecular hydrogen bond $\mathrm{O} 2{ }^{\prime} \mathrm{H}-\mathrm{O} 2$ is also possible for the $\mathrm{C}^{\prime}$ '-endo conformations of the ribose ring. The map of the potential energy surface with coordinates of $\chi$ and $\beta$ angles for Ua3 $x$ anticonformers family is presented in Fig. 5. It is clearly seen

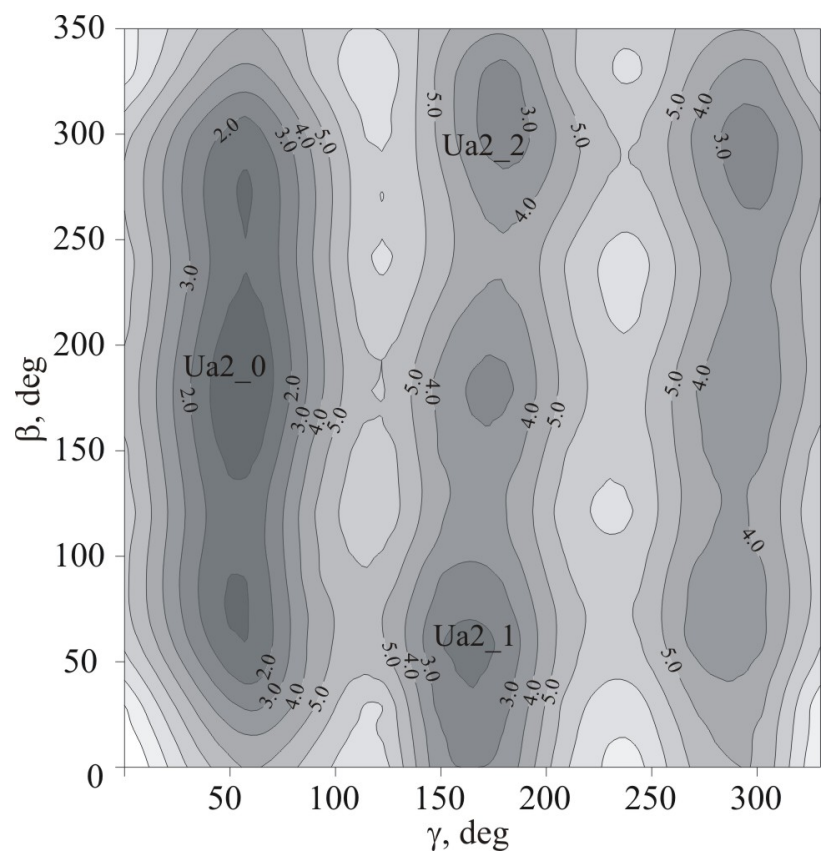

Fig. 3. Map of the potential energy surface of the anti-conformers of Ur with intramolecular hydrogen bond $\mathrm{O} 2{ }^{\prime} \mathrm{H}-\mathrm{O} 2$ and $\mathrm{C} 2$ '-endo conformation of ribose ring. The torsion angles $\beta$ and $\gamma$ were varied with step of $30^{\circ}$. The labeling of the isolines on the map is given in $\mathrm{kcal} / \mathrm{mol}$.

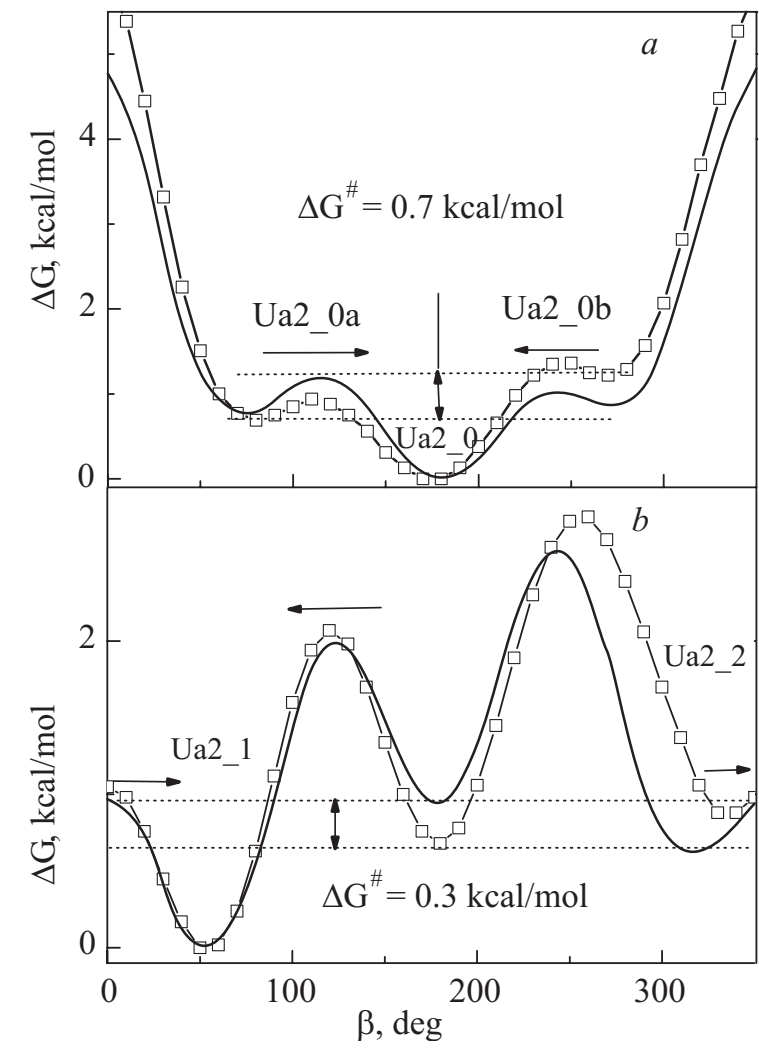

Fig. 4. Profiles of the conformational barrier calculated by the DFT/B3LYP/cc-pVDZ and MP2/cc-pVDZ methods with step of $10^{\circ}$ along the bottom of grooves for the global minimum Ua2_0 (a) and Ua2_1 conformations (b).

from Fig. 5 that positions of grooves with minima are practically in line with those in Fig. 3. In the upper right part of

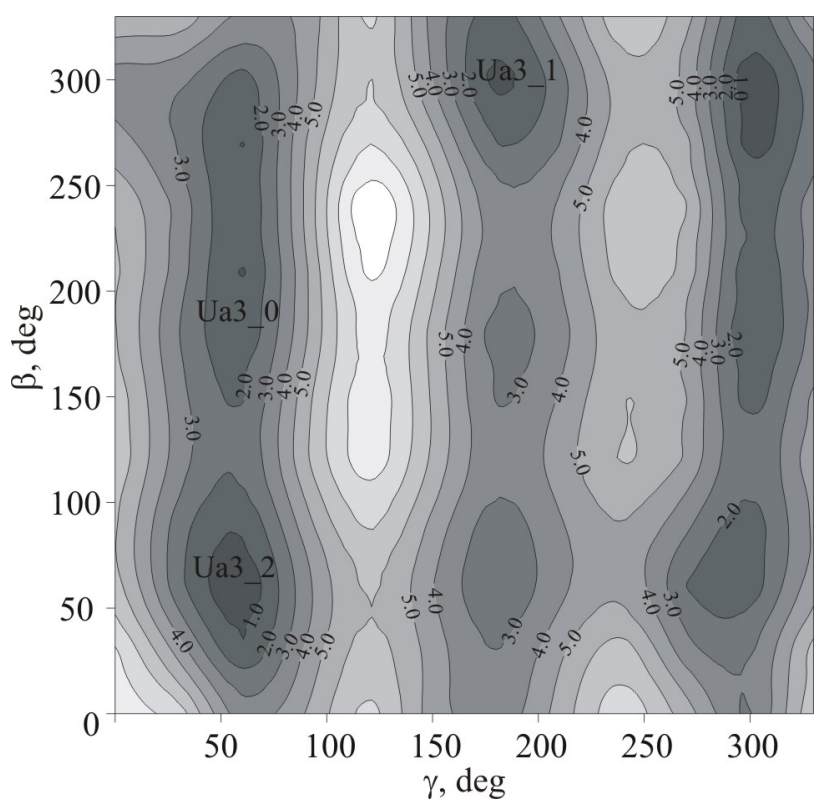

Fig. 5. Map of the potential energy surface of the anti-conformers of Ur with the intramolecular hydrogen bonds $\mathrm{O} 2{ }^{\prime} \mathrm{H}-\mathrm{O} 2$ and the C3'-endo conformation of ribose ring. The torsion angles $\beta$ and $\gamma$ were varied with step of $30^{\circ}$. 
Fig. 5 this agreement is complete, since in this region the C3'-endo structures are unstable and during the process of geometry optimization they transform to the $\mathrm{C} 2$ '-endo structure. Therefore the transitions between related C2'endo and C3'-endo structures of the family of anticonformers Uan_x are of more important than transitions between the Ua $3 \_x$ conformers on the bottom of grooves (Fig. 5). Figure 6, $a$ shows that the barrier between the conformers Ua3_2 and Ua2_0a is practically absent in the calculation with method DFT/cc-pVDZ and increases to 0.75 $\mathrm{kcal} / \mathrm{mol}$ at the MP2/cc-pVDZ level of theory. Consequently, conformer Ua3_2 will be completely transformed to onformer Ua2_0a, and further to conformer Ua2_0, which according to the calculations (see below) is the global minimum for Ur.

It is known that, according to the Bader model [28], interactions between $\mathrm{C} 6 \mathrm{H}$ and $\mathrm{O}^{\prime}$ ' may be assigned to weak hydrogen bonds [29]. If the ribose ring is C3'-endo structure, this weak bond can stabilize the pyrimidine ring in the position that would prevent a stronger intramolecular hydrogen bond $\mathrm{O} 2{ }^{\prime} \mathrm{H}-\mathrm{O} 2$. The barrier between such a structure (Ua3_0) and global minimum $\mathrm{Ua} 2 \_0$ is presented in Fig. $6, b$. The calculation by the method DFT/cc-pVDZ pre-

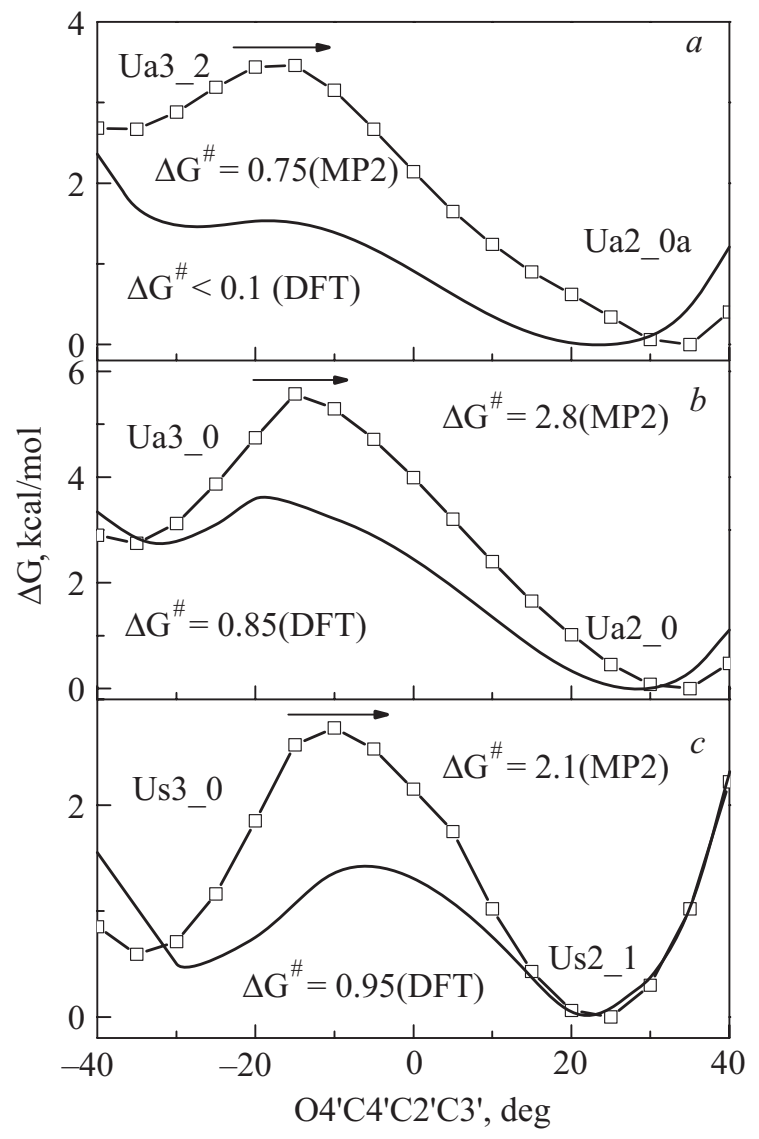

Fig. 6. The energy relaxation profiles of the conformational transitions for the different pair of anti- $(a, b)$ and syn- conformers $(c)$ calculated by the DFT/B3LYP/cc-pVDZ and MP2-cc-pVDZ methods (squares) along dihedral angle $\mathrm{O} 4^{\prime} \mathrm{C} 4{ }^{\prime} \mathrm{C} 2{ }^{\prime} \mathrm{C} 3$ ' with step of $10^{\circ}$. dicts the height of the barrier $0.85 \mathrm{kcal} / \mathrm{mol}$, but the method MP2/cc-pVPZ dramatically increases the barrier to 2.8 $\mathrm{kcal} / \mathrm{mol}$. Therefore we can reasonably assume that the conformer Ua3_0 is fixed in the Ar matrices during deposition.

Hitherto we shown that the syn-conformers of dU with the C3'-endo structure of sugar ring are not fixed in the matrix, and transforms to the $\mathrm{C} 2$ '-endo conformers [10,11]. Heights of the transition barriers calculated for these conformers were $0.7 \mathrm{kcal} / \mathrm{mol}$ (DFT/cc-pVDZ method) and 1.3 $\mathrm{kcal} / \mathrm{mol}$ (MP2/cc-pVDZ method). The calculation of the profile of the barrier for the transition between the Ur synconformers Us3_0 $\rightarrow$ Us2_1 shows a significant increases in the barrier both at the DFT/cc-pVDZ $(0.95 \mathrm{kcal} / \mathrm{mol})$, and MP2/cc-pVDZ (2.2 kcal/mol) level of theory (Fig. 6,c). Because of this, unlike dU, the syn-conformers of Ur with the C3'-endo structures of ribose may be fixed in the matrices during the process of deposition. It should be noted that, in comparison with the barriers of the $\mathrm{OH}$ group rotation (Fig. 4), the choice of the computational method (DFT or MP2) strongly influences the barrier height of C2'endo $\rightarrow \mathrm{C} 3$ '-endo transition (Fig. 5).

Based on results presented above, several structures were selected for calculations of the relative energies $\Delta E$. The characteristic structural bond angles of these conformers and the results of calculations of $\Delta E$ are presented in Table 1. The selected conformers are local minima on the potential energy surface, and it was proved by the absence of imaginary frequencies in the vibrational spectra calculated at the DFT//cc-pVDZ and DFT//aug-cc-pVDZ level of theory. Unlike dU, all Ur conformers are stable structures upon various computational methods - from the simple DFT//cc-pVDZ to the more sophisticated MP2//aug-cc-pVDZ and MP2//aug-cc-pVTZ. All the calculation methods clearly define $\mathrm{Ua} 2 \_0$ structure as global minimum (Table 1). Augmentation of the cc-pVDZ basis set with diffuse functions (aug-cc-pVDZ) markedly reduced the difference in $\Delta E$ between $\mathrm{Ua}_{2}{ }_{0}$ and the rest of conformers. It is true both for the DFT and MP2 methods. The choice of the more complicated aug-cc-pVTZ basis set slightly reduces the dispersion of $\Delta E$ values (Table 1).

Calculation of the relative free energies $\Delta G$ using Firefly program still predicts the conformer Ua2_0 as global minimum, but compared to $\Delta E$ the scatter of $\Delta G$ decreases and does not exceed $2.1 \mathrm{kcal} / \mathrm{mol}$ for all the conformers considered. For comparison with the experimental data, it is desirable to turn from $\Delta G(T)$ values to $N(T)$ occupancy. To calculate the occupancies of conformers the standard formula was used [30]:

$$
N_{j}(T)=\frac{k_{j} \exp \left[-\Delta G_{j}(T) / R T\right]}{\sum_{i=0}^{n} k_{i} \exp \left[-\Delta G_{i}(T) / R T\right]} 100 \%
$$

where $k_{j}$ is the coefficient of degeneration of $j$ th conformation. 
Table 1. Structure parameters (characteristic angles) and relative energies $(\Delta E, \Delta G)$ in $\mathrm{kcal} / \mathrm{mole}$ of the main anti- and synconformers of Ur

\begin{tabular}{|c|c|c|c|c|c|c|c|c|c|c|}
\hline \multicolumn{11}{|c|}{ Conformers } \\
\hline Structure parameters & $\mathrm{Ua} 2 \_0$ & Ua2_1 & $\mathrm{Ua} 2 \_2$ & Ua3_0 & Ua3_1 & Ua3_2 & Us3_0 & Us3_1 & Us2_0 & Us2_1 \\
\hline \multicolumn{11}{|l|}{ Torsion angles } \\
\hline$\angle \mathrm{P}$ & 205 & 214 & 202 & 17 & 64 & 63 & 43 & 41 & 159 & 159 \\
\hline$\angle \chi$ & -178 & -176 & -172 & -165 & -176 & -176 & 64 & 67 & 63 & 62 \\
\hline$\angle \gamma$ & 54 & 172 & 177 & 54 & -179 & 56 & 48 & -60 & 46 & 45 \\
\hline$\angle \beta$ & 178 & 62 & -31 & -176 & -56 & 62 & 40 & 176 & 63 & 63 \\
\hline$\angle \varepsilon$ & 146 & 146 & 148 & 81 & 91 & 92 & -140 & -56 & 155 & -82 \\
\hline \multicolumn{11}{|l|}{$\begin{array}{l}\text { Relative energies and me- } \\
\text { thods of calculation }\end{array}$} \\
\hline $\begin{array}{c}\Delta E \\
\text { DFT/cc-pVDZ }\end{array}$ & 0 & 1.38 & 1.94 & 2.76 & 2.76 & 2.19 & 2.26 & 4.33 & 0.94 & 1.76 \\
\hline $\begin{array}{c}\Delta E \\
\mathrm{MP} 2 / \mathrm{cc}-\mathrm{pVDZ}\end{array}$ & 0 & 1.57 & 2.45 & 2.70 & 4.20 & 3.51 & 2.70 & 4.26 & 1.51 & 2.07 \\
\hline $\begin{array}{c}\Delta E \\
\text { DFT/aug-cc-pVDZ }\end{array}$ & 0 & 1.53 & 1.64 & 1.48 & 1.50 & 1.63 & 1.69 & 2.65 & 0.88 & 1.25 \\
\hline $\begin{array}{c}\Delta E \\
\text { DFT/aug-cc-pVTZ }\end{array}$ & 0 & 1.88 & 1.57 & 1.57 & 2.26 & 1.82 & 1.51 & 2.57 & 0.75 & 1.07 \\
\hline $\begin{array}{c}\Delta E \\
\text { MP2/aug-cc-pVDZ }\end{array}$ & 0 & 1.62 & 2.75 & 1.81 & 3.34 & 3.12 & 2.04 & 3.45 & 1.00 & 1.71 \\
\hline $\begin{array}{c}\Delta E \\
\mathrm{MP} \text { 2/aug-cc-pVTZ }\end{array}$ & 0 & 1.51 & & 1.91 & & & 1.94 & & 0.79 & \\
\hline $\begin{array}{c}\Delta E \\
\text { MP4/aug-cc-pVDZ// } \\
\text { MP2/aug-cc-pVDZ }\end{array}$ & 0 & 1.56 & & 1.96 & & & 1.74 & & 0.79 & 1.49 \\
\hline $\begin{array}{c}\Delta \mathrm{G}(420 \mathrm{~K}) \\
\text { aug-cc-pVDZ* }\end{array}$ & 0 & 1.44 & 0.82 & 1.02 & 1.15 & 1.31 & 1.90 & 2.0 & 1.78 & 2.09 \\
\hline $\begin{array}{c}\text { Occupancies in gas at } \\
420 \mathrm{~K}, \%\end{array}$ & $44.1 * *$ & 4.8 & $20.2 * *$ & 7.9 & 6.8 & 5.6 & 2.8 & 2.5 & 3.2 & 2.2 \\
\hline $\begin{array}{l}\text { Occupancies in matrix by } \\
\text { calculations }\end{array}$ & 56.5 & 25 & 0 & 7.9 & 0 & 0 & 2.8 & 2.5 & 3.2 & 2.2 \\
\hline $\begin{array}{c}\text { Occupancies in matrix by } \\
\text { experiment }\end{array}$ & 10 & 20 & 0 & 30 & 0 & 0 & 20 & 0 & 10 & 10 \\
\hline
\end{tabular}

Comment: ${ }^{*}-\Delta E$ from calculation by the DFT/B3LYP/aug-cc-pVDZ method; ${ }^{* *}$ — with taking into account the conformations Ua2_0a, Ua2_0b, and two-fold degeneracy of the Ua2_2 conformer.

Calculation results at the temperature $T=420 \mathrm{~K}$ are presented in Table 1. These calculations take into account two-fold degeneracy of the Ua2_2 conformer (Fig. 4,b) and the presence of the Ua2 $0 \mathrm{a}$ and $\mathrm{Ua} 2$ _ $\mathrm{b}$ conformers (Fig. 4,a) in the gas phase with total occupancy being $17.1 \%$. As was discussed above, during deposition in the matrix the Ua2_0a, Ua2_0b and Ua3_2 conformers transform into Ua2_0. It increases the Ua2_0 occupancy to $56.5 \%$. In the same way, the Ua2_2 conformer transforms to Ua2_1 and occupancy of this conformer increases to $25 \%$. Occupancies of other five conformers is different from zero and are in the range from 2.5 to $8 \%$. Table 2 presents frequencies of characteristic spectral bands of seven conformers that could be fixed in the matrix. As the characteristic band of Us3_1 is absent in the range near $3605 \mathrm{~cm}^{-1}$ of the experimental spectrum, its bands are replaced by Ua3_1 ones (Table 2). It is evident that many characteristic bands are overlapped and additional information from the experimental spectra is necessary for the assignment of these bands. In order to obtain experimental evidences for the above discussion, we compared the spectra of dU and Ur, as well as the shift of conformational equilibrium by UV irradiation of matrices and their annealing. The UV irradiation of matrix samples permits to increase of the intensities of bands of the low populated conformers. But the effect of the UV irradiation is insignificant if the conformers have a small difference of occupations in matrix. Most likely, in this case the irradia- 
Table 2. Experimental (Ar matrices) and calculated (DFT/B3LYP/aug-cc-pVDZ) frequencies and intensities of the bands of vibrational spectra of the Ur main conformers

\begin{tabular}{|c|c|c|c|c|c|c|c|c|c|c|c|c|c|c|c|c|c|c|}
\hline \multicolumn{19}{|c|}{ Conformer } \\
\hline \multirow[t]{2}{*}{$\begin{array}{l}\text { Vibrational } \\
\text { mode }\end{array}$} & \multicolumn{2}{|c|}{$\begin{array}{l}\mathrm{dU} \text { in } \mathrm{Ar} \\
\text { matrix }\end{array}$} & \multicolumn{2}{|c|}{$\begin{array}{l}\text { Ur in Ar } \\
\text { matrix }\end{array}$} & \multicolumn{2}{|c|}{ Ua2_0 } & \multicolumn{2}{|c|}{ Ua2_1 } & \multicolumn{2}{|c|}{ Ua3_0 } & \multicolumn{2}{|c|}{ Ua3_1 } & \multicolumn{2}{|c|}{ Us3_0 } & \multicolumn{2}{|c|}{ Us2_0 } & \multicolumn{2}{|c|}{ Us2_1 } \\
\hline & $\mathrm{cm}^{-1}$ & $I$ & $\mathrm{~cm}^{-1}$ & $I$ & $\mathrm{~cm}^{-1}$ & $I$ & $\mathrm{~cm}^{-1}$ & $I^{a}$ & $\mathrm{~cm}^{-1}$ & $I^{a}$ & $\mathrm{~cm}^{-1}$ & $I^{a}$ & $\mathrm{~cm}^{-1}$ & $I^{a}$ & $\mathrm{~cm}^{-1}$ & $I^{a}$ & $\mathrm{~cm}^{-1}$ & $I^{a}$ \\
\hline$v \mathrm{O}^{\prime} \mathrm{H}$ & 3664 & 3.4 & 3665 & 2.4 & 3657 & 47 & 3626 & 37 & 3665 & 51 & 3634 & 29 & & & & & & \\
\hline$v \mathrm{O} 2$ 'H & 3641 & 1.9 & 3642 & 0.9 & & & & & 3642 & 66 & & & 3660 & 68 & 3662 & 65 & 3665 & 66 \\
\hline $\begin{array}{l}v \text { hb_O3'H } \\
v \text { hb_O5'H }\end{array}$ & $\begin{array}{l}3624 \\
3611 \\
3597 \\
\end{array}$ & 1.2 & & & & & & & & & & & & & & & & \\
\hline $\begin{array}{l}v \text { hb_O3'H } \\
v \text { hb_O2'H } \\
v \text { hb_O5'H }\end{array}$ & & & $\begin{array}{l}3584 \\
3560 \\
3551 \\
\end{array}$ & 4 & 3553 & 69 & 3564 & 67 & 3579 & 64 & $\begin{array}{l}3565 \\
3548\end{array}$ & $\begin{array}{c}58 \\
178\end{array}$ & $\begin{array}{l}3529 \\
3556\end{array}$ & $\begin{array}{l}102 \\
213\end{array}$ & 3579 & 51 & 3567 & 78 \\
\hline$v$ hb_O5'H & $\begin{array}{l}3491 \\
3485\end{array}$ & 4.7 & $\begin{array}{l}3493 \\
3485 \\
3477 \\
\end{array}$ & 5.9 & & & & & & & & & & & 3493 & 426 & 3476 & 488 \\
\hline$v$ hb_O2'H & & & $\begin{array}{l}3458 \\
3449\end{array}$ & 2.4 & 3451 & 351 & 3464 & 303 & & & & & & & & & & \\
\hline$v \mathrm{~N} 3 \mathrm{H}$ & 3428 & 6.4 & 3427 & 6.4 & 3418 & 81 & 3418 & 78 & 3422 & 69 & 3417 & 77 & 3417 & 69 & 3415 & 72 & 3416 & 73 \\
\hline $\begin{array}{l}v(\mathrm{C} 2=\mathrm{O}) \\
v(\mathrm{C} 4=\mathrm{O})\end{array}$ & & 88 & & 84 & & & & & & & & & & & & & & \\
\hline
\end{tabular}

Comment: $I$ is the relative integrated intensity; $I^{a}$ is the absolute integrated intensity, $\mathrm{km} / \mathrm{mol}$; vhb_OH is the hydroxyl group participating in the intramolecular hydrogen bond. The scaling of the calculated frequencies was done according to the Ar matrix with a multiplier $=0.9535$.

tion-induced changes in the band intensity should depend on the relative resistance of conformers in matrix to the $\mathrm{UV}$ irradiation. As the dU molecules, Ur ones partially decompose in the Ar matrix under the UV irradiation. After irradiation the intensities of all bands decreases distinctly, with the exception of the band at $3605 \mathrm{~cm}^{-1}$, which may be assigned to vibrations of the $\mathrm{O}^{\prime} 3 \mathrm{H}$ and $\mathrm{O}^{\prime}{ }^{\prime} \mathrm{H}$ groups (Fig. 7). Therefore we may assume that for Ur realized scenario with several close-populated conformers, rather than dominance of structure Ua2_0.

If the barrier is close to $2 \mathrm{kcal} / \mathrm{mol}$, the annealing of the matrix may provide useful information about the transitions between the conformers. In Fig. 7 is shown that annealing leads to redistribution of intensities between two groups of bands. This is evidence of the transition between the $s y n$-conformers Us3_0 $\rightarrow$ Us $2 \_1$ and hence the presence Us3_0 in the matrix. As the transition barrier of Ua3_0 $\rightarrow$ Ua2 $\_0$ is of close height, the presence of Ua3_0 in the matrix can be considered as well-provided (it should be noted that this conformation has no well-defined characteristic bands). For the remaining observed bands a slight decrease in intensities connected with the partial association of monomeric molecules (Fig. 7). Given the difference in the absolute intensities of the bands (Table 2), the population of Us 3 _ 0 practically coincides with the

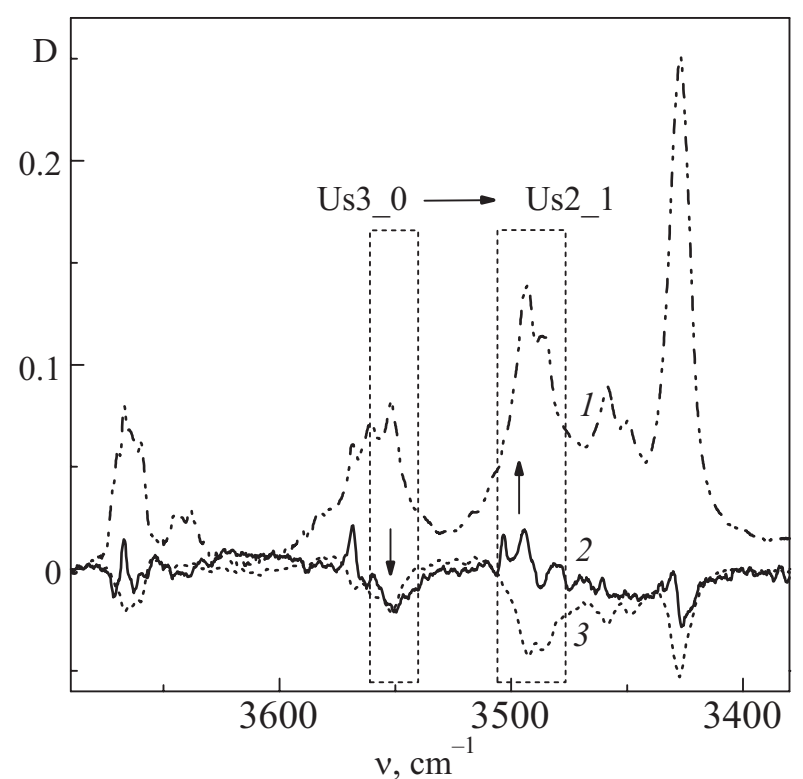

Fig. 7. Effect of UV irradiation and annealing of matrix on the occupancies of the Ur conformers in the Ar matrix $(T=6 \mathrm{~K}$, Matrix to sample ratio $M / S=700)$ in the $v \mathrm{OH}, v \mathrm{NH}$ region $(3690-3390$ $\mathrm{cm}^{-1}$ ). Spectrum after deposition of matrix (1); difference spectrum after annealing of matrix at ( $T=29 \mathrm{~K}, t=5 \mathrm{~min})(2)$; difference spectrum after UV irradiation $(\lambda=200-300 \mathrm{~nm}, t=20 \mathrm{~min})(3)$. 
population of the Us2_0 conformers. Unlike dU, characteristic bands of structures with the hydrogen bonds $\mathrm{O}^{\prime} \mathrm{H}-$ O3' and O3'H-O5' (like to the Us3_1 conformer) were not practically found in the experimental spectra.

We showed earlier that frequencies and intensities of the $v \mathrm{~N} 3 \mathrm{H}$ stretching vibration have a small difference for 2-dU conformers $[10,11]$. This statement is also true for $\mathrm{Ur}$ conformers (Table 2). Therefore, for the correct comparison of the spectra, experimental intensities of $\mathrm{dU}$ and $\mathrm{Ur}$ spectra are brought to the equal intensity of the $v \mathrm{~N} 3 \mathrm{H}$ bands (Table 2). The conclusion may be inferred that the growth of occupancy of the syn-conformers of $\mathrm{Ur}$ is based on the decreasing of intensity of the band $3665 \mathrm{~cm}^{-1}$ and increasing intensity of the absorption nearly $3485 \mathrm{~cm}^{-1}$. Besides, now Us3_0 structure can be added to uridine synconformers (see discussion above). As well, in Ur spectra the intensity of $3642 \mathrm{~cm}^{-1}$ band lowers, belonging to the free vibrations $\mathrm{O}^{\prime} \mathrm{H}^{\prime}\left(\mathrm{O}^{\prime} 3 \mathrm{H}\right)$. All this evidences the common domination of Ur syn- and anti-conformers with the intramolecular hydrogen bonds $\mathrm{O} 5^{\prime} \mathrm{H}-\mathrm{O} 2$ and $\mathrm{O} 2^{\prime} \mathrm{H}-\mathrm{O} 2$, respectively.

For the experimental estimation (Table 1) of occupancies of the conformers in matrices the standard method [11] was implemented by using the calculated and experimental intensities of the characteristic bands and decomposition of the complicated contours to Gaussian functions. The validity of this estimation was supported by the comparison of the experimental and synthesized spectra. The program SYNSPEC [31] was applied for the synthesis of vibrational spectra by using the Gaussian contours with full width on half maximum of $10 \mathrm{~cm}^{-1}$. If the calculated conformer occupancy is applied (Table 1), the synthetic spectrum (Fig. 8) differs markedly from the experimental one. But the experimental estimation of the conformer occupancy leads to the qualitative agreement between the synthesized and experimental spectra (Fig. 8). It is obvious that calculations overestimate the Ua2_0 conformer occupancy. The most exact methods MP4/aug-ccpVDZ//MP2/aug-cc-pVDZ and MP2/aug-cc-pVTZ do not change essentially the $\Delta E$ and $\Delta G$, distribution respectively (Table 1). Most likely, the discrepancy between calculated and experimental data is connected with anharmonicity of uridine low-frequency vibrations but not with inaccuracy of electronic energy calculations.

Because of overlapping of spectral bands of some conformers, the accuracy of their occupancy in the matrices is rather low (worse than $\pm 10 \%$ ). Perhaps method of the «burning out» of low barrier conformers in the supersonic beam of Ar before the deposition of matrices [26,32] hold promise for the simplifying of experimental spectra and improving of accuracy.

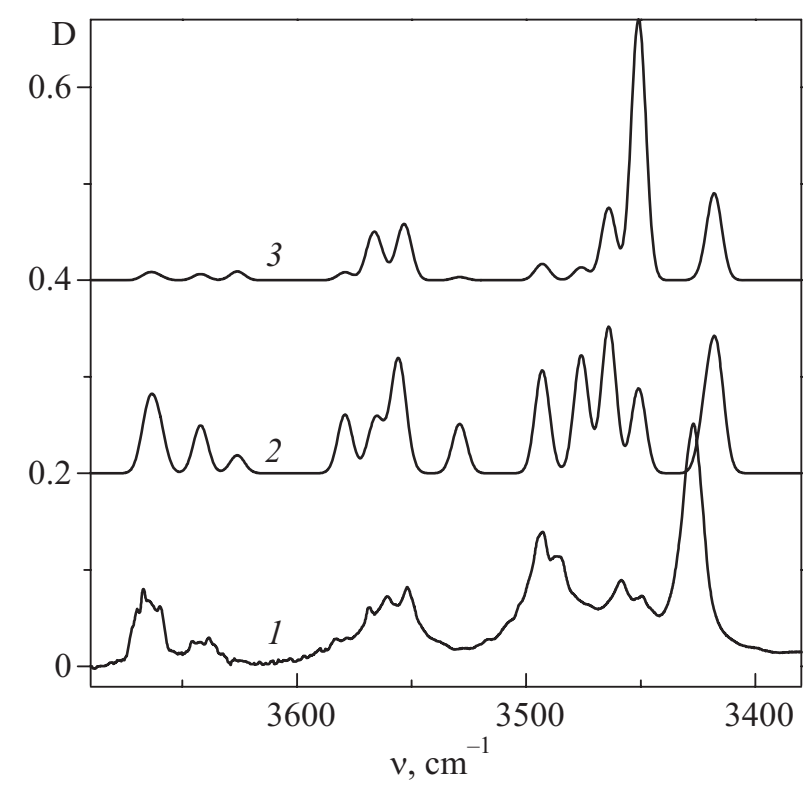

Fig. 8. The comparison of the experimental spectrum and spectra which were synthesized by program SYNSPEC [30]. All frequencies and intensities were used from the Table 2. The experimental spectrum (1); spectrum was synthesized according the experimental estimation of the occupancies of the Ur conformers (Table 1); spectrum was synthesized according the computational estimation of the occupancies of the Ur conformers (Table 1) (3).

\section{Conclusions}

By the methods of low temperature matrix isolation and quantum chemical calculation it was shown that conformers of Ur included the intramolecular hydrogen bonds with participation of the carbonyl group $(\mathrm{C} 2=\mathrm{O})$ are dominated $(70 \pm 10) \%$ in Ar matrices at $12 \mathrm{~K}$.

The comparison of Ur spectra with the spectra of $\mathrm{dU}$ shown the growth of occupancies of the syn-conformers with the intramolecular hydrogen bond $\mathrm{O}^{3}{ }^{\prime} \mathrm{H}-\mathrm{O} 2$ up to $(40 \pm 10) \%$. Mainly, it arises from appearance of the Us3_o conformer with the $\mathrm{C} 3$ '-endo structure of the ribose ring in the matrix. The populations of the syn-conformers are close to the anti-conformers with intramolecular hydrogen bonds $\mathrm{O} 2{ }^{\prime} \mathrm{H}-\mathrm{O} 2$ and $\mathrm{C}^{2}$ '-endo structures of ribose which is dominated for both types of conformers.

The results of quantum chemical calculations of the relative free energies agreed satisfactorily with low temperature experimental data, excepting anti-conformer Ua2_0.

The present work was carried out due to the financial support of the National Academy of Sciences of Ukraine. The author thanks S.G. Stepanian for helpful discussion and L.F. Belous for his great help in organization and carrying out of calculations on the GRID-cluster of B. Verkin Institute for Low Temperature Physics and Engineering of the National Academy of Sciences of Ukraine. 
1. F. Piuzzi, M. Mons, I. Dimicoli, B. Tardivel, and Q. Zhao, Chem. Phys. 270, 205 (2001).

2. R. Weinkauf, J.-P. Schermann, M.S. de Vries, and K. Kleinermanns, Eur. Phys. J. D20, 309 (2002).

3. R.N. Casaes, J.B. Paul, R.P. McLaughlin, R.J. Saykally, and T. van Mourik, J. Phys. Chem. A108, 10989 (2004).

4. J.P. Simons, R.A. Jockusch, P. Carcabal, I.H. Nig, R.T. Kroemer, N.A. MacLeod, and L.C. Snoek, Int. Rev. Phys. Chem. 24, 489 (2005).

5. M.Y. Choi and R.E. Miller, J. Phys. Chem. A111, 2475 (2007).

6. S. Cradock and A.J. Hinchliffe, Matrix Isolation, Cambridge University Press, New York (1975).

7. S.A. Krasnokutski, A.Yu. Ivanov, V. Izvekov, G.G. Sheina, and Yu.P. Blagoi, J. Mol. Struct. 482-483, 249 (1998).

8. A.Yu. Ivanov, S.A. Krasnokutski, G. Sheina, and Yu.P. Blagoi, Spectrochim. Acta A59, 1959 (2003).

9. A.Yu. Ivanov, S.A. Krasnokutski, and G.G. Sheina, Fiz. Nizk. Temp. 29, 1065 (2003) [Low Temp. Phys. 29, 809 (2003)].

10. A.Yu. Ivanov and V.A. Karachevtsev, Fiz. Nizk. Temp. 33, 772 (2007) [Low Temp. Phys. 33, 590 (2007)].

11. A.Yu. Ivanov. Fiz. Nizk. Temp. 34, 962 (2008) [Low Temp. Phys. 34, 762 (2008)].

12. M.W. Powner, B. Berland, and J.D. Sutherland, Nature 459, 239, (2009).

13. W. Saenger, Principles of Nucleic Acids Structure, SpringerVerlag, New York (1984).

14. N. Leulliot, M. Ghomi, G. Scalmani, and G. Berthier. J. Phys. Chem. A103, 8716 (1999).

15. N. Leulliot, M. Ghomi, H. Jobic, O. Bouloussa, V. Baumruk, and C. Coulombeau, J. Phys. Chem. B103, 10934 (1999).

16. M.-P. Gaigeot, N. Leulliot, M. Ghomi, H. Jobic, C. Coulombeau, and O. Bouloussa, Chem. Phys. 261, 217 (2000).
17. A. Hocquet, N. Leulliot, and M. Ghomi. J. Phys. Chem. B104, 4560 (2000).

18. R.O. Zhurakivsky and D.M. Hovorun, Biopolymers and Cell. 24, 142 (2008).

19. A.Yu. Ivanov, A.M. Plokhotnichenko, E.D. Radchenko, G.G. Sheina, and Yu.P. Blagoi, J. Mol. Struct. 372, 91 (1995).

20. A. Kovacs and A.Yu. Ivanov, J. Phys. Chem. B113, 2151 (2009).

21. A.Yu. Ivanov and A.M. Plokhotnichenko, Instr. Experim. Techn. 52, 308 (2009).

22. Alex A. Granovsky, Firefly Version 7.1.G, http://classic.chem.msu.su/gran/firefly/index.html (2009).

23. M.W. Schmidt, K.K. Baldridge, J.A. Boatz, S.T. Elbert, M.S. Gordon, J.H. Jensen, S. Koseki, N. Matsunaga, K.A. Nguyen, S. Su, T.L. Windus, M. Dupuis, and J.A. Montgomery, J. Comput. Chem. 14, 1347 (1993).

24. T.H. Dunning, Jr., J. Chem. Phys. 90, 1007 (1989).

25. M. Wojdyr. Program FITYK 0.8.1, http://www.unipress.waw.pl/fityk/ (2008).

26. P. Felder and Hs.H. Gunthard, Chem. Phys. 71, 9 (1982).

27. A.J. Barnes, J. Mol. Struct. 113, 161 (1984).

28. R.F.W. Bader, Atoms in Molecules. A Quantum Theory, Clarendon, Oxford (1990).

29. S.J. Grabowski, J. Phys. Chem. A105, 10739 (2001).

30. J.H. Jensen and M.S. Gordon J. Am. Chem. Soc. 113, 7917 (1991).

31. K. Irikura, Program SYNSPEC, National Institute of Standards and Technology, Gaithersburg, MD 20899, USA (1995).

32. V. Vidya, K. Sankaran, and K.S. Viswanathan, Chem. Phys. Lett. 258, 113 (1996). 